\title{
Distinct contribution of IL-6, TNF- $\alpha$, IL-1, and IL-10 to T cell-mediated spontaneous autoimmune arthritis in mice
}

\author{
Hiroshi Hata,1,2 Noriko Sakaguchi,,1,3 Hiroyuki Yoshitomi,,1,2 Yoichiroh Iwakura,4 \\ Kenji Sekikawa, ${ }^{5}$ Yoshiaki Azuma, ${ }^{6}$ Chieko Kanai,7 Eiko Moriizumi,7 \\ Takashi Nomura, ${ }^{1}$ Takashi Nakamura, ${ }^{2}$ and Shimon Sakaguchi1,3,8

\begin{abstract}
1Department of Experimental Pathology, Institute for Frontier Medical Sciences, Kyoto University, Kyoto, Japan. ${ }^{2}$ Department of Orthopedic Surgery, Faculty of Medicine, Kyoto University, Kyoto, Japan. ${ }^{3}$ Laboratory for Immunopathology, RIKEN Research Center for Allergy and Immunology, Yokohama, Japan. ${ }^{4}$ nstitute of Medical Sciences, Tokyo University, Tokyo, Japan. ${ }^{5}$ Department of Molecular Biology and Immunology, National Institute of Agrobiological Sciences, Tsukuba, Ibaraki, Japan. ${ }^{6}$ Teijin Institute for Biomedical Research, Teijin Pharma Ltd., Tokyo, Japan. ${ }^{7}$ Tokyo Metropolitan Institute of Gerontology, Tokyo, Japan. ${ }^{8}$ Core Research for Evolutional Science and Technology (CREST), Japan Science and Technology Agency, Kawaguchi, Japan.
\end{abstract}

\begin{abstract}
Cytokines play key roles in spontaneous $\mathrm{CD}^{+} \mathrm{T}$ cell-mediated chronic autoimmune arthritis in SKG mice, a new model of rheumatoid arthritis. Genetic deficiency in IL-6 completely suppressed the development of arthritis in SKG mice, irrespective of the persistence of circulating rheumatoid factor. Either IL-1 or TNF- $\alpha$ deficiency retarded the onset of arthritis and substantially reduced its incidence and severity. IL-10 deficien$c y$, on the other hand, exacerbated disease, whereas IL-4 or IFN- $\gamma$ deficiency did not alter the disease course. Synovial fluid of arthritic SKG mice contained high amounts of IL-6, TNF- $\alpha$, and IL-1, in accord with active transcription of these cytokine genes in the afflicted joints. Notably, immunohistochemistry revealed that distinct subsets of synovial cells produced different cytokines in the inflamed synovium: the superficial synovial lining cells mainly produced IL-1 and TNF- $\alpha$, whereas scattered subsynovial cells produced IL-6. Thus, IL-6, IL-1, TNF- $\alpha$, and IL-10 play distinct roles in the development of SKG arthritis; arthritogenic CD4 ${ }^{+}$T cells are not required to skew to either Th1 or Th2; and the appearance of rheumatoid factor is independent of joint inflammation. The results also indicate that targeting not only each cytokine but also each cell population secreting distinct cytokines could be an effective treatment of rheumatoid arthritis.
\end{abstract}

\section{Introduction}

Rheumatoid arthritis (RA) is a chronic systemic inflammatory disease of unknown etiology that primarily affects the synovial membranes of multiple joints (1). A cardinal feature of joint inflammation in RA is proliferative inflammation of synovial cells, i.e., synovitis, which results in the destruction of the adjacent cartilage and bone. Although $\mathrm{CD}^{+} \mathrm{T}$ cells are currently assumed to be the prime mediators of synovitis, it remains obscure how arthritogenic $\mathrm{CD}^{+} \mathrm{T}$ cells activate synovial cells to proliferate or, upon activation, how the autonomous proliferation of synovial cells is maintained, leading to the destruction of the joint (1). It has been well documented that cytokines play indispensable roles in these processes (2). TNF- $\alpha$ and IL-6, for example, contribute to joint inflammation in RA, as illustrated by the effects of neutralizing TNF- $\alpha$ or blocking IL- 6 receptor to ameliorate RA $(3,4)$. IFN- $\gamma$, IL-4, and IL-10 formed by Th1 or Th2 CD4+ $\mathrm{T}$ cells may also participate in synovitis, as observed in other autoimmune diseases $(1,2)$. The way in which these cytokines contribute to the development of arthritides including RA, however, is a subject of controversy, as cytokines exert different effects in different models of arthritis.

Nonstandard abbreviations used: $\zeta$-associated protein of $70 \mathrm{kDa}$ (ZAP-70); collagen-induced arthritis (CIA); rheumatoid arthritis (RA); rheumatoid factor (RF); Src homology 2 (SH2).

Conflict of interest: The authors have declared that no conflict of interest exists.

Citation for this article: J. Clin. Invest. 114:582-588 (2004).

doi:10.1172/JCI200421795.
For example, IL-6 deficiency variously results in exacerbation of, amelioration of, or no effects on arthritis, depending on the particular model (5-9). Neutralization of TNF- $\alpha$ has different effects in collagen-induced arthritis (CIA) and streptococcal cell wallinduced arthritis (10). IFN- $\gamma$ may not only mediate Th1 responses in arthritis but also suppress the destruction of cartilage and bone by inhibiting the generation of osteoclasts (11). Furthermore, it is unclear how relevant the available arthritis models are to human RA. For example, CIA, one of the most widely used models of RA, is not accompanied by the appearance of rheumatoid factor (RF), which is present in about $70 \%$ of RA patients (1). In this report, we have analyzed the contribution of pro- and anti-inflammatory cytokines to the spontaneous development and chronic progression of $\mathrm{CD}^{+} \mathrm{T}$ cell-mediated autoimmune arthritis in a newly established mouse model of RA.

SKG mice spontaneously develop T cell-mediated chronic autoimmune arthritis as a consequence of a mutation of the gene encoding an Src homology 2 (SH2) domain of $\zeta$-associated protein of $70 \mathrm{kDa}$ (ZAP-70), a key signal transduction molecule in T cells (12). This mutation impairs positive and negative selection of $\mathrm{T}$ cells in the thymus, leading to thymic production of arthritogenic $\mathrm{T}$ cells. Clinically, joint swelling begins in small joints of the digits, progressing in a symmetrical fashion to larger joints including wrists and ankles. Histologically, the swollen joints show severe synovitis with formation of pannus invading and eroding adjacent cartilage and subchondral bone. SKG mice develop extra-articular lesions, such as interstitial pneumonitis, vasculitides, and subcuta- 
neous necrobiotic nodules not unlike rheumatoid nodules in RA. Serologically, they develop high titers of RF and autoantibodies specific for type II collagen. Furthermore, $\mathrm{CD}^{+} \mathrm{T}$ cells can adoptively transfer arthritis in SKG mice, which have a BALB/c genetic background, to T cell-deficient BALB/c nude or T cell/B cell-deficient SCID mice, which indicates that the disease is a T cell-mediated autoimmune disease. In addition to the causative gene, the polymorphism of the $M H C$ gene also contributes to the occurrence of SKG arthritis depending on environmental conditions. Thus, this spontaneous autoimmune arthritis in mice resembles human RA in clinical and histological characteristics of articular and extra-articular lesions, in serological characteristics, and in the key role of $\mathrm{CD}^{+} \mathrm{T}$ cells in initiating arthritis (12).

In contrast to other organ-specific autoimmune diseases in which self-reactive $\mathrm{T}$ cells destroy the target cells (e.g., insulinsecreting $\beta$ cells in type 1 diabetes mellitus), a key feature of SKG autoimmune arthritis, and human RA for that matter, is that $\mathrm{T}$ cells do not destroy but stimulate synoviocytes to proliferate and invade the surrounding cartilage and bone. The selective development of arthritis in SKG mice, despite their general alteration in the $\mathrm{T}$ cell repertoire, could be attributed at least in part to a high sensitivity of synoviocytes to immunological stimuli, including $\mathrm{T}$ cell self-reactivity, due to their immunologically unique features. The synoviocytes are, for example, intrinsically capable of producing proinflammatory cytokines and matrix metalloproteinases; are composed of type A macrophage-like and type B fibroblast-like synoviocytes, both of which are highly sensitive to various immunological stimuli including cytokines; and are devoid of basement membrane and tight junctions, allowing their easy invasion to the surrounding tissue (1). We have therefore analyzed in this report how the synoviocytes stimulated by arthritogenic $\mathrm{CD}^{+} \mathrm{T}$ cells mediate arthritis in SKG mice and how those cytokines produced by the stimulated synoviocytes or arthritogenic $\mathrm{CD} 4^{+} \mathrm{T}$ cells contribute to the development and progression of arthritis.

\section{Results}

Clinical and histological features of SKG arthritis. In SKG mice, joint swelling began to develop in a few digits around 2 months of age, progressing to other digits and to larger joints (wrists and ankles) in a symmetrical fashion (Supplemental Figure 1; supplemental material available at http://www.jci.org/cgi/ content/full/114/4/582/DC1). Histology of swollen joints showed vigorous proliferation of synovial cells, resulting in pannus formation and infiltration of mononuclear cells and neutrophils to the subsynovial region (Supplemental Figure 2). Pannus-destroyed cartilage and bone showed the appearance of multinuclear osteoclasts at the interface between invading synovial tissue and the adjacent cartilage and subchondral bone. Synoviocytes that formed a few layers of superficial lining of pannus were characteristically tall and plump and bore a large cytoplasm, as shown by H\&E staining. Electron microscopy revealed that the majority of these superficial lining cells were type B fibroblast-like synoviocytes with occasional type A macrophage-like synoviocytes (Supplemental Figure 3). It also showed infiltration of lymphocytes and neutrophils to the sublining region and some synoviocytes apparently in apoptosis, as has been observed in human RA (13). In 8-month-old SKG mice, microcomputerized tomography revealed erosion of the cartilage and subchondral bone in knee joints (Supplemental Figure 4).
Thus, SKG mice develop severe proliferative synovitis accompanying destruction of cartilage and subchondral bone of digits, wrists, ankles, and knees, as do humans with RA (1).

Immunohistochemistry of SKG synovitis. Immunohistochemical staining of synovial tissues of 4-month-old SKG mice with various antibodies revealed the following. A number of $\mathrm{CD}^{+}$ $\mathrm{T}$ cells, but few $\mathrm{CD}^{+} \mathrm{T}$ cells, infiltrated the sublining tissue, where B cells also formed aggregates (Figure 1, A-C). Granulocytes (Figure 1D) and macrophages (Figure 1E) were abundant in the joint cavity and also subsynovial region. The distribution of macrophages corresponded to that of class II MHC-expressing cells (Figure 1F). Many cells expressing CD49d (also known as very late antigen 4; VLA-4) infiltrated the subsynovia (Figure $1 \mathrm{G}$ ), and its ligand VCAM-1 was expressed in vascular endothelial cells and interstitial cells (Figure $1 \mathrm{H}$ ), which indicates recruitment of lymphocytes to the inflamed synovial tissue (Figure 1I) (14). Toluidine blue staining revealed few mast cells in the inflamed tissue (data not shown).

Cytokine production in arthritic joints. Ankle joints of the majority of 24-week-old SKG mice with severe joint swelling exhibited highlevel expression of IL-1 $\beta$ and IL- 6 mRNA, as assessed by real-time RT-PCR; half of the mice also actively transcribed TNF- $\alpha$ mRNA (Figure 2A). In contrast, 8-week-old SKG mice without apparent joint swelling showed no IL- 6 transcription and only a slight expression of IL- $1 \beta$, although TNF- $\alpha$ mRNA was detected in some young SKG mice. IL-2, IL-4, IL-10, and IFN- $\gamma$ mRNA messages were not detected even in 24-week-old SKG mice (data not shown).

The joint fluid taken from swollen ankle joints of 32-week-old SKG mice contained high amounts of IL-1 $\beta$, IL- 6 , and TNF- $\alpha$ (Figure $2 B$ ). Serum levels of these cytokines were below detectable levels by ELISA in the majority of mice, although $20 \%$ of them bore low but significant amounts of IL-6 in their sera (data not shown).

Immunohistochemical staining of inflamed synovial tissue of SKG mice revealed distinct staining patterns of TNF- $\alpha$ and IL-1 $\beta$ versus IL-6. Cells expressing IL-1 $\beta$ and TNF- $\alpha$ were detected almost exclusively in one or two layers of hypertrophic superficial lining cells facing the joint cavity (Figure 2, C and D). In contrast, cells expressing IL-6 were smaller than the superficial
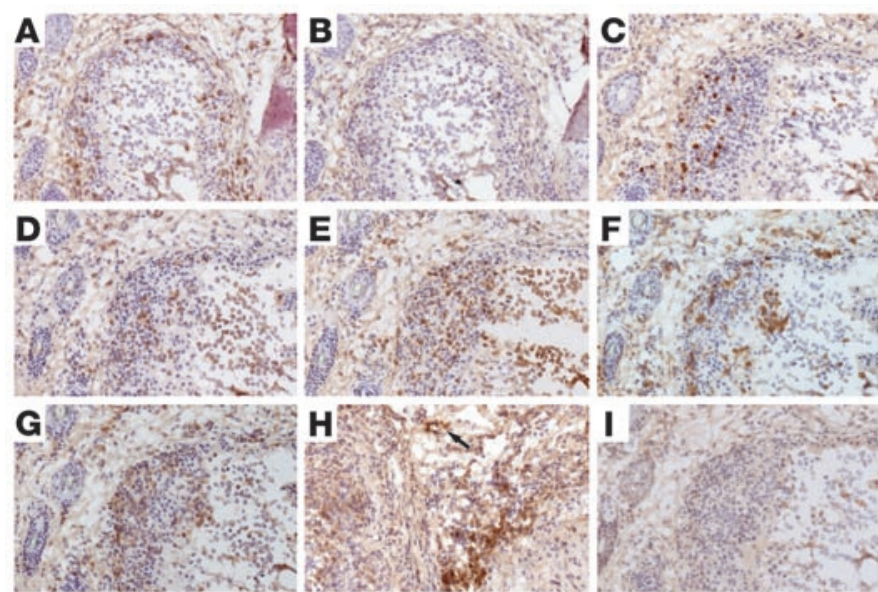

Figure 1

Immunohistology of synovitis. Serial sections of a finger joint of a 5-monthold SKG mouse were stained for CD4 (A), CD8 (B), B220 (C), Gr-1 (D), CD11b (E), I-A/I-E (F), CD49d (G), or VCAM-1 (H) with staining control (I). Arrow indicates vasculature. Original magnification, $\times 20$. 

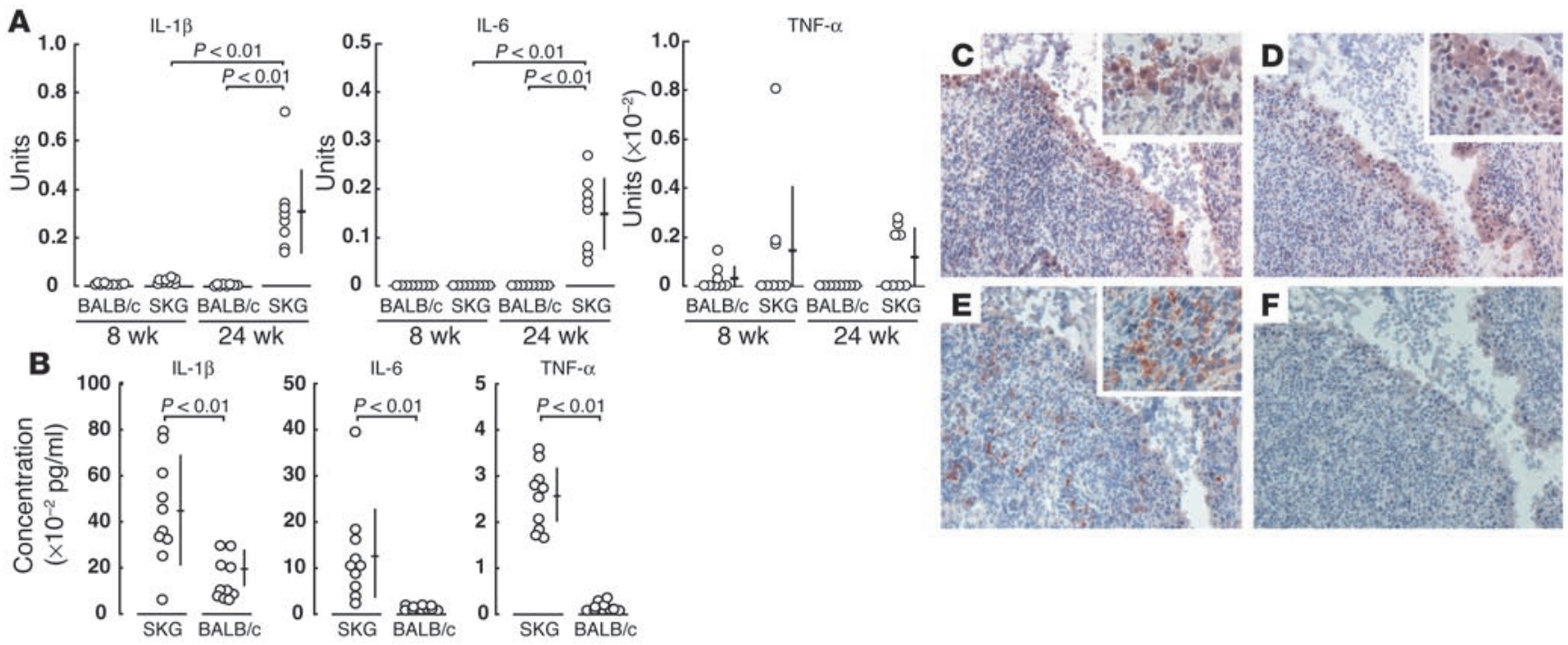

Figure 2

Expression of cytokines at mRNA and protein levels. (A) Quantitative RT-PCR for indicated genes was performed with ankle joints from 8- or 24-week-old SKG or BALB/c mice. Bars show the means \pm SD. See Methods for the definition of units. (B) Concentrations of indicated cytokines, assessed by ELISA, in the joint fluid of 32-week-old SKG and BALB/c mice ( $n=10$ each). Bars show the means \pm SD. (C-F) Immunohistochemical staining of the synovial tissue of a finger joint of a 4-month-old SKG mouse for IL-1 $\beta$ (C), TNF- $\alpha$ (D), or IL-6 (E), with staining control (F) (original magnification, $\times 40)$. Insets show higher magnifications of a part of synovium.

lining cells and scattered in the sublining region (Figure 2E). The localization of IL-6-expressing cells correlated with that of MAC-1 $1^{+}$I-A/I-E $E^{+}$macrophages or CD4 $4^{+}$cells (Figure 1).

These results collectively indicate that the inflamed synovial tissue of SKG mice actively produces IL- $1 \beta$, TNF- $\alpha$ and IL- 6 ; the cells forming TNF- $\alpha$ and IL- $1 \beta$ are apparently the same and constitute the superficial lining layer; and the cells mainly forming IL- 6 are apparently different from those forming IL- $1 \beta$ and TNF- $\alpha$ and localize differently.

Effect of deficiency in particular cytokines on the development and progression of SKG arthritis. To determine the roles of proinflammatory cytokines IL- $1 \alpha$, IL-1 $\beta$, TNF- $\alpha$, and IL- 6 in the development of SKG arthritis, homozygously cytokine gene-deficient (-/-), heterozygously cytokine gene-deficient $(+/-)$, or cytokine gene-intact $(+/+)$ female SKG mice were prepared and maintained in our conventional animal facility, where the majority of cytokine gene-intact female SKG mice began to exhibit joint swelling at 11 to 12 weeks of age (Figure 3A). Severity of arthritis in these mice varied from swelling of some joints of the digits to marked swelling of large joints (the average score was $3.6 \pm 1.5$; see Methods for clinical assessment of arthritis) at 32 weeks of age. Histology of swollen joints of 32-week-old SKG mice revealed severe synovitis and destruction of cartilage and bone (Figure 3B). Notably, IL-6-/-SKG mice showed no joint swelling macroscopically, no histologically evident synovitis, and no destruction of cartilage and bone (Figure 3C). Some IL- $1 \alpha / \beta^{-/-}$SKG mice started to develop arthritis around 15 weeks of age with a significantly lower incidence than IL-1 $\alpha / \beta^{+/+}$SKG mice. Although the incidence in IL-1//- mice was $60 \%$ at 32 weeks of age, severity was very low, with joint swelling restricted to only 1 or 2 joints of the digits. These swollen joints showed histologically evident synovitis but destruction of cartilage and bone was generally less severe than in cytokine-intact SKG mice (data not shown). These results were similar in the TNF- $\alpha^{-/-}$SKG mice: the onset was delayed, incidence lowered to $20 \%$ of controls, and severity substantially reduced to swelling of a single joint of a digit (see legend to Figure 3A for statistical analyses of the data).

Interstitial pneumonitis - which was observed in SKG mice older than 8 months (12) - among these cytokine-deficient mice was too mild in severity and low in incidence at 32 weeks of age for comparisons to be made (data not shown).

IFN- $\gamma$-deficient SKG mice developed arthritis with no statistically significant differences in severity and incidence from IFN- $\gamma^{+/-}$ or IFN $-\gamma^{+/+}$mice during 24 weeks of observation, although IFN- $\gamma^{-/-}$ SKG mice displayed progressive weight loss and died earlier as compared with wild-type controls maintained under the same conditions (12). We did not observe significant difference in histology between IFN- $\gamma^{-/}$and IFN- $\gamma^{+/-}$or IFN- $\gamma^{+/+}$mice, although the IFN- $\gamma^{-/-}$ mice, when they became debilitated, generally showed lesser degrees of arthritis compared with the IFN- $\gamma^{+/-}$or IFN- $\gamma^{+/+}$mice (data not shown). IL-4-/- SKG mice, on the other hand, survived well to 32 weeks of age; the time of onset, incidence and severity of arthritis were not significantly different among IL-4-/, IL-4+/-, or IL-4+/+ mice. Thus, IFN- $\gamma$ and IL- 4 are apparently dispensable for the development of SKG arthritis.

IL-10-/- SKG mice showed a consistently higher incidence of disease and mean arthritis score than IL-10+/- or IL- $10^{+/+}$SKG mice, although the susceptibility to colitis of IL-10-/- mice restricted the period of observation to 24 weeks (15). Histological examination of $\mathrm{IL}-10^{-/-}$mice that had survived to this age showed severe synovitis accompanying joint destruction (Figure 3D). Thus, IL-10 is suppressive for the development of SKG arthritis.

In these experiments, SKG mice with heterozygous deficiency in IL-1, TNF- $\alpha$, IL-6, or IL-10 showed incidences and severities of arthritis that were intermediate between those of homozygously cytokine-deficient and cytokine-intact mice. This result indicates that the disease-enhancing or -protective effects of the cytokines are dose dependent. 
A
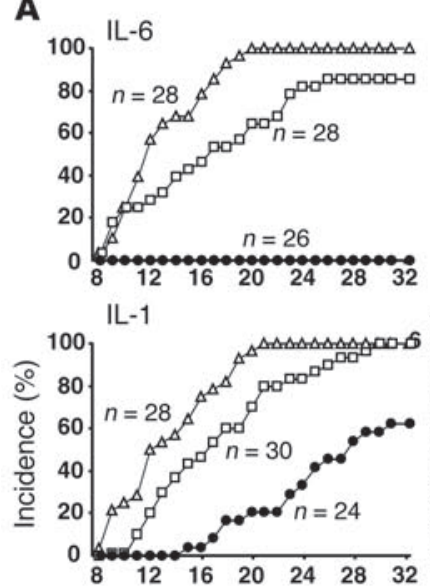

TNF- $\alpha$

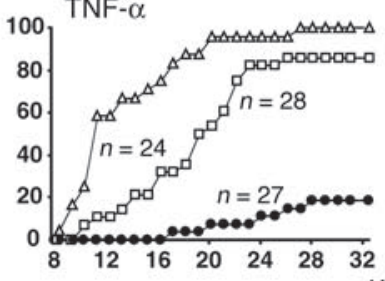

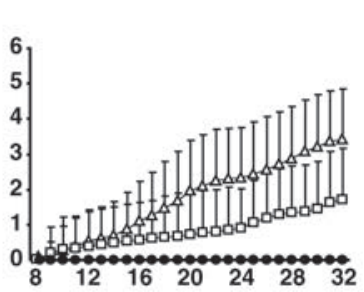
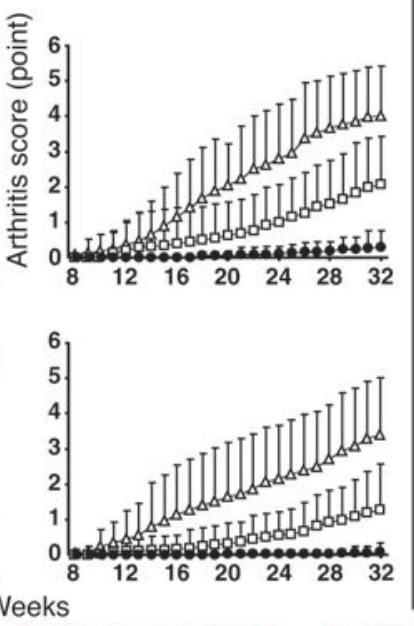
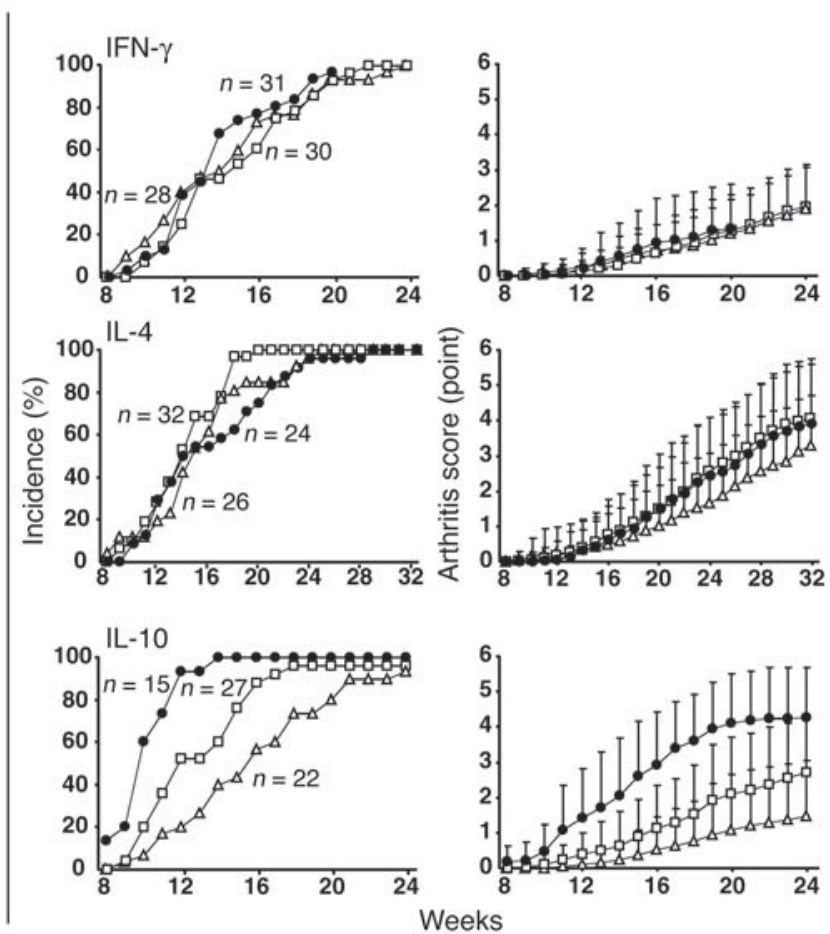

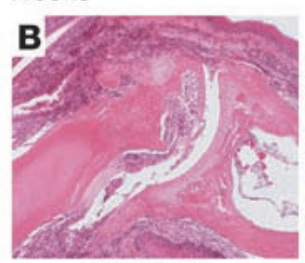

WT SKG

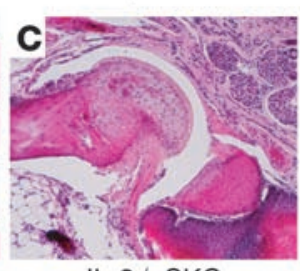

IL-6--SKG

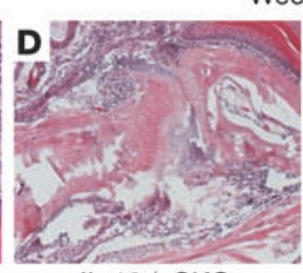

$\mathrm{IL}-10^{-1-} \mathrm{SKG}$

Figure 3

Development of arthritis in cytokine-deficient SKG mice. (A) Incidence and severity of arthritis in female SKG mice homozygously (-/-) (filled circles) or heterozygously (+/-) (open squares) deficient in indicated cytokines or cytokine-intact $(+/+)$ (open triangles). Vertical bars represent the means $\pm \mathrm{SD}$ of the whole group of mice. Arthritis scores are significantly different $(P<0.01)$ between IL- $6^{+/+}$and IL-6 $6^{+/-}$mice at $19-32$ weeks;

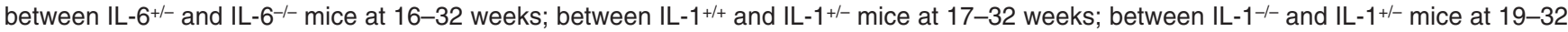
weeks; between TNF- $\alpha^{+/+}$and TNF- $\alpha^{+/-}$mice at 15-32 weeks; between TNF- $\alpha^{-/-}$and TNF- $\alpha^{+/-}$mice at 21-32 weeks; between IL-10 IL-10+/- mice at 18-24 weeks; and between IL-10-/- and IL-10+/- mice at 12-24 weeks. (B-D) Histology of finger joints of a 32-week-old wild-type (B) or IL-6-deficient (C) or a 24-week-old IL-10-deficient SKG mouse (D) (original magnification, $\times 10$ ).

$R F$ and joint inflammation. The IL-6-, TNF- $\alpha-$, or IL-1-deficient mice developed high titers of IgM-type RF even in the absence of joint inflammation (Figure 4A). This indicates that the development of IgM-RF is independent of joint inflammation. The result contrasted with the development of IgG anti-type II collagen autoantibody in arthritic mice but not in nonarthritic IL-6-deficient mice, which suggests the possible roles of anti-type II collagen antibody as an active mediator of joint inflammation or a product of joint destruction (Figure 4B).

\section{Discussion}

This study showed that proliferative synovitis with pannus formation and resultant joint destruction in SKG mice are immunopathologically similar to those observed in human RA and that, despite the pleiotropy, redundancy, and cross-regulation in cytokine functions, deficiency in either IL-6, IL-1, or TNF- $\alpha$ can inhibit the development and progression of SKG arthritis, which is apparently independent of the development of RF.
It is well documented in human RA and in animal models that IL-1, IL- 6 , and TNF- $\alpha$ synergistically mediate synovitis and destruction of cartilage and bone (1-3, 5, 6, 16-19), that IL- 1 and TNF- $\alpha$ trigger the secretion of IL- 6 by synovial cells (2), and that anti-TNF- $\alpha$ treatment can lower IL-6 formation $(20,21)$. It is still unclear, however, how the formation and action of these cytokines are controlled at molecular and cellular levels (22-24). Here we showed that IL-6 is apparently produced by subsynovial stromal cells (macrophages and DCs; Figure 1) and presumably some CD4 $4^{+} \mathrm{T}$ cells, whereas IL- 1 and TNF- $\alpha$ are mainly produced by superficial lining cells facing the joint cavity and producing matrix metalloproteinases as also shown in other arthritis models and RA (25-28). This difference in cellular sources between IL- 6 and IL-1/TNF- $\alpha$ indicates that not only neutralization of these cytokines or blockade of their actions at a molecular level, but also reduction in the number of the stromal cells producing each cytokine or modifying their functions may ameliorate arthritis.

TNF- $\alpha$ deficiency inhibited arthritis effectively but not completely in SKG mice; i.e., the onset was delayed, incidence lowered 
A

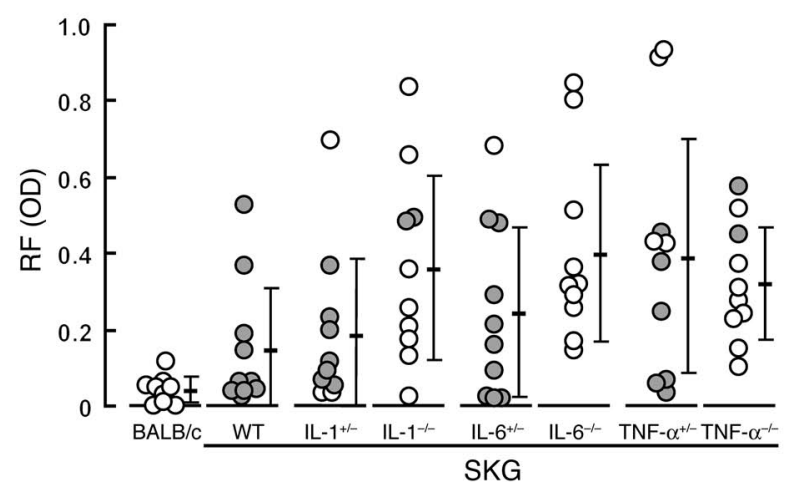

B

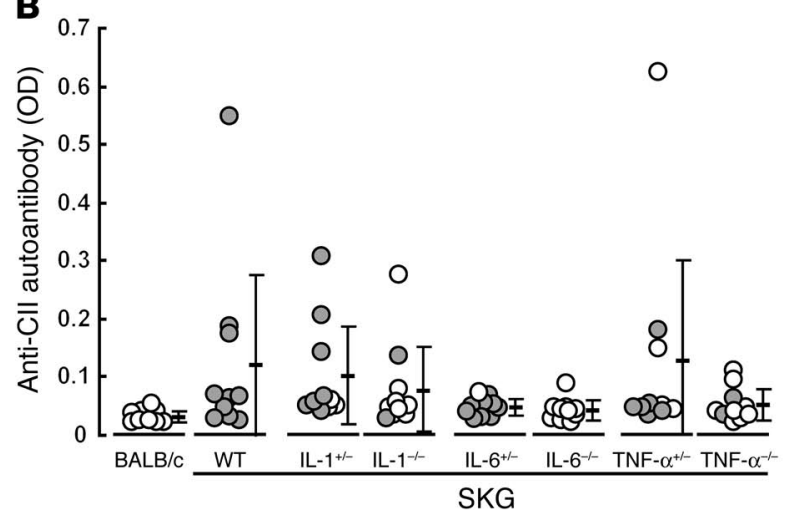

Figure 4

Autoantibodies in cytokine-deficient SKG mice. RF (A) or anti-type II collagen (anti-CII) autoantibody titers (B) in 32-week-old SKG mice homozygously or heterozygously deficient in indicated cytokines, with wild-type SKG mice and age-matched BALB/c mice as controls. Filled circles indicate mice with joint swelling (arthritis score $\geq 1.0$ ); open circles indicate mice without joint swelling (arthritis score $<1.0)$. First 10 mice that had reached 32 weeks of age in each group shown in Figure 3 were analyzed.

to $20 \%$ of controls, and severity substantially reduced to swelling of a single joint of a digit. It can be argued that the MHC haplotype of TNF- $\alpha$-deficient SKG mice could influence the development of arthritis, since TNF- $\alpha$-deficient SKG mice bear an H-2b haplotype in contrast with SKG mice, which bear an $\mathrm{H}-2 \mathrm{~d}$ haplotype. This is because the founder strain of TNF- $\alpha$ deficiency was of $\mathrm{H}-2 \mathrm{~b}$ haplotype, and the TNF- $\alpha$ locus, which is closely linked with the MHC locus, was cosegregated with the MHC in establishing the congenic TNF- $\alpha$-deficient SKG mice (29). We previously showed that the MHC haplotype of SKG mice can influence the incidence and severity of arthritis (e.g., H-2d haplotype confers susceptibility to arthritis in SKG mice [ref. 12]). It is unlikely, however, that the H-2b haplotype of TNF- $\alpha$-deficient SKG mice alters the incidence and severity of arthritis, because SKG mice on BALB.B (H-2b) background showed an incidence and severity of arthritis similar to those of SKG mice (our unpublished data). Thus, TNF- $\alpha$ deficiency per se can inhibit the development of arthritis. On the other hand, the failure of TNF- $\alpha$ deficiency to completely inhibit arthritis suggests that proinflammatory cytokine pathways that spare TNF- $\alpha$ may also be operative in SKG arthritis. This substantial but incomplete inhibition of SKG arthritis by TNF- $\alpha$ deficiency may have a common basis with the clinical result that only about $30-40 \%$ of RA patients show dramatic responses to TNF inhibitors (30).

It is well documented in arthritis that pro- or anti-inflammatory cytokines facilitate or inhibit activation of various types of cells, including macrophages/monocytes, neutrophils, and T cells, which are involved in local inflammation (16). Another function of cytokines may be to alter the degree of $\mathrm{T}$ cell-mediated control of autoimmune $\mathrm{T}$ cells, thereby allowing arthritogenic $\mathrm{T}$ cells to chronically mediate arthritis. For example, IL-10 not only suppresses the formation of TNF- $\alpha$ and IL- 6 by macrophages, synoviocytes, and T cells, as shown in other experimental models (31-33), but also may mediate or augment $\mathrm{CD} 25^{+} \mathrm{CD} 4^{+} \mathrm{T}$ cell-mediated immunoregulation, as shown in other autoimmune/inflammatory diseases $(34,35)$. IL-10 deficiency may therefore attenuate the $\mathrm{T}$ cell-mediated suppression on arthritogenic $\mathrm{T}$ cells, thus contributing to the exacerbation of arthritis in SKG mice. Furthermore, in addition to its local and systemic proinflammatory effects $(20,36)$, IL- 6 may render arthritogenic $\mathrm{T}$ cells refractory to the suppressive control exerted by natu- rally arising $\mathrm{CD} 25^{+} \mathrm{CD} 4^{+}$regulatory $\mathrm{T}$ cells $(37) . \mathrm{CD} 25^{+} \mathrm{CD} 4^{+} \mathrm{T}$ cells, derived from normal BALB/c mice, indeed prevented SKG arthritis effectively when the animals were inoculated before onset of the disease, as observed in CIA (ref. 38 and our unpublished data). We are currently investigating whether IL-6 deficiency rectifies the susceptibility of arthritogenic $\mathrm{T}$ cells to the $\mathrm{CD} 25^{+} \mathrm{CD} 4^{+} \mathrm{T}$ cell-mediated suppression and thereby contributes to inhibiting the development of SKG arthritis. Whether TNF- $\alpha$ or IL- 1 can affect T cell-mediated immunoregulation by altering the functions of APCs or regulatory T cells also remains to be determined (39).

Although $\mathrm{CD}^{+} \mathrm{T}$ cells play pivotal roles in SKG arthritis, they show impaired activation and proliferation upon $\mathrm{T}$ cell receptor stimulation because of a structurally altered ZAP-70 and resulting alteration in $\mathrm{T}$ cell signal transduction in SKG T cells (12). Despite this defect, SKG T cells are competent in secreting various cytokines, including IL-2, IL-4, IL-10, TNF- $\alpha$, and IFN- $\gamma$, upon PMA/ionomycin stimulation, in a pattern of cytokine production and kinetics similar to that of BALB/c T cells (Supplemental Figure $5 \mathrm{~A}$ ). $\mathrm{CD}^{+} \mathrm{T}$ cells from young SKG mice even produced slightly higher amounts of IFN- $\gamma$, IL-4, and IL-10 and smaller amounts of TNF- $\alpha$ than BALB/c CD $4^{+}$T cells (Supplemental Figure 5B). Moreover, there was no significant alteration of the disease course in SKG mice with IFN- $\gamma$ or IL-4 deficiency, which is in contrast with other models $(40,41)$. These findings taken together indicate that SKG CD4 ${ }^{+} \mathrm{T}$ cells are not committed to Th1 or Th 2 cells, or TNF- $\alpha$ production, due to the altered signal transduction through ZAP-70; skewing of CD $4^{+}$arthritogenic T cells to either Th1 or Th2 is not absolutely required for the development and/or progression of arthritis; and cross-regulation, if any, by Th1 or Th2 cells is not significant in inhibiting arthritis in this model. It is possible that $\mathrm{T}$ cells in SKG mice trigger the activation and proliferation of synoviocytes in a cell-contact manner or via other cytokines (42). Notably, a large amount of mRNA for IL-17, which is secreted by activated T cells and able to stimulate synovial cells (43), can be detected in the joints of arthritic SKG mice (our unpublished data).

The disease in SKG mice is a chronic systemic inflammatory disease like human RA $(1,12)$. For example, SKG mice spontaneously develop RF as a systemic manifestation of the disease. An intriguing finding in cytokine-deficient SKG mice is that they developed high 
titers of RF irrespective of whether arthritis was inhibited or not. For example, IL-6-deficient mice with no arthritis developed titers of RF equivalent to those of SKG mice. This cannot be attributed to hypergammaglobulinemia in SKG mice, because SKG mice without arthritis did not develop anti-DNA autoantibodies or anti-type II collagen autoantibodies, which may mediate joint inflammation or develop as a consequence of joint destruction (Figure 4) (12). Furthermore, transfer of thymocytes alone from SKG mice produced severe arthritis in SCID mice, which suggests that B cells may not be an absolute requirement for elicitation and progression of arthritis in this model (12). These results taken together indicate that RF is independent of joint inflammation per se in SKG mice. It is known, on the other hand, that RA patients with RF undergo a more aggressive and destructive course of arthritis (1). Considering that RF also develops in various other inflammatory diseases including microbial infections, it is likely that RF may not be a primary mediator of arthritis but may secondarily enhance joint inflammation, for example, by forming immune complexes that deposit in the joint $(44,45)$.

In conclusion, cytokines play essential roles in SKG arthritis in a manner similar to that in human RA. SKG mice can be instrumental for further studying the contribution of pro- and anti-inflammatory cytokines to the development and progression of RA, for elucidating how arthritogenic $T$ cells stimulate synoviocytes to cause arthritis, and for devising effective treatment of the disease.

\section{Methods}

Mice. IL-1 $\alpha / \beta-$, TNF- $\alpha-$, or IL- $6-$ deficient mice (IL- $1^{-/-}, \mathrm{TNF}^{-\alpha^{-/}}$, and IL- $6^{-/}$, respectively) were backcrossed to BALB/c mice more than 8 times (29). BALB/ c IL-10-deficient (IL-10-/-) mice were kindly provided by D. Rennick (DNAX Institute, Palo Alto, California, USA) (15). BALB/c IFN- $\gamma^{-/-}$and IL-4/mice were purchased from The Jackson Laboratory (Bar Harbor, Maine, USA) $(46,47)$. These cytokine-deficient BALB/c mice were backcrossed three times to SKG mice, which have the BALB/c genetic background (12). Such Skg-homozygous but cytokine gene-heterozygous mice were intercrossed, and resulting female $\mathrm{Skg} / \mathrm{Skg}$ littermates with homozygously or heterozygously cytokine-deficient or cytokine-intact female mice were used in the present study. All mice were maintained in our animal facility under conventional microbial conditions. All experiments were conducted according to the institutional guidelines for animal welfare of the Institute for Frontier Medical Sciences at Kyoto University.

Clinical assessment of SKG arthritis. Joint swelling was monitored by inspection and scored as follows: 0 , no joint swelling; 0.1 , swelling of one finger joint; 0.5 , mild swelling of wrist or ankle; 1.0 , severe swelling of wrist or ankle. Scores for all digits, wrists, and ankles were totaled for each mouse (12).

Antibodies. The following antibodies were used: anti-CD4 (H129.19), anti-CD8a (53-6.7), anti-CD11b (M1/70), anti-CD45RB/B220 (RA3-6B2), anti-CD49d (9C10), anti-CD106 (429), and anti-I-A/I-E (M5/114.15.2), purchased from BD Biosciences - Pharmingen (San Diego, California, USA); anti-IL-1 $\beta$ (AB-401-NA) (R\&D Systems Inc., Minneapolis, Minnesota, USA); anti-TNF- $\alpha$ (L-19) (Santa Cruz Biotechnology Inc., Santa Cruz, California, USA); anti-IL-6 (D97) (Innogenetics, Gent, Belgium). For intracellular cytokine detection, the following mAb's were used: anti-IFN- $\gamma$ (XMG1.2), anti-TNF- $\alpha$ (MP6-XT22), anti-IL-2 (JES6-5H4), anti-IL-4 (11B11), and anti-IL-10 (JES5-16E3) (BD Biosciences - Pharmingen).

$R T-P C R$. Total RNA was extracted from ankle joints using Isogen reagent (Nippon Gene Co., Tokyo, Japan) and reverse transcribed using Superscript II (Invitrogen Japan K.K., Tokyo, Japan). IL-1 $\beta$, IL-6, or TNF- $\alpha$ mRNA levels were quantified by real-time PCR using QuantiTect Assay (Qiagen K.K., Tokyo, Japan) and normalized by hypoxanthine-guanin phosphoribosyl transferase (HPRT) as previously described (48). The quantities of these cytokine mRNAs were expressed as units by defining the levels of each cytokine mRNA in J774.1, a BALB/c macrophage cell line, stimulated with $1 \mu \mathrm{g} / \mathrm{ml}$ LPS for 24 hours, as one unit.

Histology and immunohistochemistry. Joints were fixed in $10 \%$ formalin, decalcified by $10 \%$ EDTA in PBS for 3 days, embedded in paraffin, sectioned, and stained with hematoxylin and eosin. For immunohistochemical staining, cryostat section of digits were fixed in cold acetone for 10 minutes, washed in PBS, and depleted of endogenous peroxidase by treatment with $0.3 \% \mathrm{H}_{2} \mathrm{O}_{2}$ in absolute methanol for 15 minutes. After blocking nonspecific binding with $10 \%$ normal rabbit serum in PBS for 30 minutes, the sections were incubated with primary mAb's at appropriate dilutions for 1 hour at room temperature, washed, incubated with biotinylated rabbit anti-rat IgG pre-adsorbed with rabbit serum, washed and incubated with avidinbiotinylated horseradish peroxidase complex $(\mathrm{ABC})$ and diaminobenzidine tetrahydrochloride (DAB) (Elite kit; Vector Laboratories Inc., Burlingame, California, USA), and counterstained with Mayer's hematoxylin.

For immunohistochemical staining of cytokines, sections were fixed for 15 minutes in ice-cold $4 \%$ phosphate-buffered paraformaldehyde ( $\mathrm{pH}$ 7.4). The sections were then washed in HEPES-buffered saline solution (HEPES-BSS) (pH 7.1, Gibco; Invitrogen Corp., Carlsbad, California, USA) supplemented with $0.1 \%$ saponin (Sigma-Aldrich, St. Louis, Missouri, USA). All further incubations and washes were carried out using BSS-saponin. Endogenous peroxidase was inactivated by treatment with $1 \%$ formalin in BSS-saponin for 2 minutes, followed by sodium azide $(0.1 \mathrm{M})$ containing $0.3 \% \mathrm{H}_{2} \mathrm{O}_{2}$ for 30 minutes, blocked with $20 \%$ normal rabbit serum for 60 minutes, incubated overnight at $4^{\circ} \mathrm{C}$ with anti-cytokine $\mathrm{mAb}$ 's at appropriate dilutions. The sections were then incubated with the secondary antibodies and $\mathrm{ABC}$, as described above. Color was developed with AEC (DakoCytomation, Carpinteria, California, USA), and the sections were counterstained with Mayer's hematoxylin.

ELISA for autoantibody. Affinity-purified mouse IgG $(5 \mu \mathrm{g} / \mathrm{ml})$ and $10 \mu \mathrm{g} / \mathrm{ml}$ of bovine type II collagen (Funakoshi Co. Ltd., Tokyo, Japan) in PBS (pH 7.2) were used for overnight coating of ELISA plates (ICN Biomedicals Inc., Aurora, Ohio, USA). Test sera were diluted to 1:10 for anti-type II collagen antibody or 1:20 for RF assay. Alkaline phosphatase conjugated anti-mouse IgG or IgM (for RF assay) (Southern Biotechnology Associates Inc., Birmingham, Alabama, USA) was used at $1 \mu \mathrm{g} / \mathrm{ml}$ as the secondary reagent. Titer of RF and anti-type II collagen antibody was expressed as optical density (49).

ELISA for cytokines. The capsules of ankle joints were cut open, joint cavity was washed with $5 \mu \mathrm{l} \mathrm{PBS}$, and $1 \mu \mathrm{l}$ of withdrawn joint fluid was diluted 10 -fold to assess the concentrations of IL-1 $\beta$, IL- 6 , and TNF- $\alpha$ by ELISA (Biosource International, Camarillo, California, USA) according to the manufacturer's instruction, with the detection limits of $7 \mathrm{pg} / \mathrm{ml}$ for IL-1, $3 \mathrm{pg} / \mathrm{ml}$ for IL- 6 , and $3 \mathrm{pg} / \mathrm{ml}$ for TNF- $\alpha$.

Statistics. Student's $t$ test was used for statistical analyses.

\section{Acknowledgments}

This work was supported by grants-in-aid from the Ministry of Education, Science, Sports, and Culture, the Ministry of Human Welfare, and the Japan Science and Technology Agency. We thank Zoltan Fehervari for critical reading of the manuscript.

Received for publication April 5, 2004, and accepted in revised form June 29, 2004.

Address correspondence to: Shimon Sakaguchi, Department of Experimental Pathology, Institute for Frontier Medical Sciences, Kyoto University, 53 Shogoin Kawahara-cho, Sakyo-ku, Kyoto 606-8507, Japan. Phone: 81-75-751-3888; Fax: 81-75-751-3820; E-mail:shimon@frontier.kyoto-u.ac.jp. 
1. Harris, E.D. 1997. Rheumatoid arthritis. W.B. Saunders. Philadelphia, Pennsylvania, USA. 417 pP.

2. Feldmann, M., Brennan, F.M., and Maini, R.N. 1996 Role of cytokines in rheumatoid arthritis. Annu. Rev Immunol. 14:397-440.

3. Choy, E.H., et al. 2002. Therapeutic benefit of blocking interleukin- 6 activity with an anti-interleukin- 6 receptor monoclonal antibody in rheumatoid arthritis: a randomized, double-blind, placebo-controlled, dose-escalation trial. Arthritis Rheum. 46:3143-3150.

4. Elliott, M.J., et al. 1993. Treatment of rheumatoid arthritis with chimeric monoclonal antibodies to tumor necrosis factor alpha. Arthritis Rheum. 36:1681-1690.

5. van de Loo, F.A., Kuiper, S., van Enckevort, F.H., Arntz, O.J., and van den Berg, W.B. 1997. Interleukin6 reduces cartilage destruction during experimental arthritis. A study in interleukin-6-deficient mice. Am. J. Pathol. 151:177-191.

6. Sasai, M., et al. 1999. Delayed onset and reduced severity of collagen-induced arthritis in interleukin6-deficient mice. Arthritis Rheum. 42:1635-1643.

7. Anguita, J., et al. 1998. Borrelia burgdorferi-infected, interleukin-6-deficient mice have decreased Th2 responses and increased lyme arthritis. J. Infect. Dis. 178:1512-1515.

8. Alonzi, T., et al. 1998. Interleukin 6 is required for the development of collagen-induced arthritis. J. Exp. Med. 187:461-468.

9. Ji, H., et al. 2002. Critical roles for interleukin 1 and tumor necrosis factor alpha in antibody-induced arthritis. J. Exp. Med. 196:77-85.

10. Joosten, L.A., Helsen, M.M., van de Loo, F.A., and van den Berg, W.B. 1996. Anticytokine treatment of established type II collagen-induced arthritis in DBA/1 mice. A comparative study using anti-TNF alpha, anti-IL-1 alpha/beta, and IL-1Ra. Arthritis Rheum. 39:797-809.

11. Takayanagi, H., et al. 2000. T-cell-mediated regulation of osteoclastogenesis by signalling crosstalk between RANKL and IFN-gamma. Nature. 408:600-605

12. Sakaguchi, N., et al. 2003. Altered thymic T-cell selection due to a mutation of the ZAP-70 gene causes autoimmune arthritis in mice. Nature. 426:454-460.

13. Firestein, G.S., Yeo, M., and Zvaifler, N.J. 1995 Apoptosis in rheumatoid arthritis synovium. J. Clin. Invest. 96:1631-1638.

14. van Dinther-Janssen, A.C., et al. 1991. The VLA-4/ VCAM-1 pathway is involved in lymphocyte adhesion to endothelium in rheumatoid synovium. J. Immunol. 147:4207-4210.

15. Kuhn, R., Lohler, J., Rennick, D., Rajewsky, K., and Muller, W. 1993. Interleukin-10-deficient mice develop chronic enterocolitis. Cell. 75:263-274.

16. Arend, W.P. 2001. Physiology of cytokine pathways in rheumatoid arthritis. Arthritis Care Res. 45:101-106

17. Probert, L., Plows, D., Kontogeorgos, G., and Kollias, G. 1995. The type I interleukin-1 receptor acts in series with tumor necrosis factor (TNF) to induce arthritis in TNF-transgenic mice. Eur. J. Immunol. 25:1794-1797.

18. Dayer, J.M., Beutler, B., and Cerami, A. 1985
Cachectin/tumor necrosis factor stimulates collagenase and prostaglandin E2 production by human synovial cells and dermal fibroblasts. J. Exp. Med. 162:2163-2168.

19. Miyasaka, N., et al. 1988. Augmented interleukin1 production and HLA-DR expression in the synovium of rheumatoid arthritis patients. Possible involvement in joint destruction. Arthritis Rheum. 31:480-486.

20. Guerne, P.A., Zuraw, B.L., Vaughan, J.H., Carson, D.A., and Lotz, M. 1989. Synovium as a source of interleukin 6 in vitro. Contribution to local and systemic manifestations of arthritis. J. Clin. Invest. 83:585-592.

21. Charles, P., et al. 1999. Regulation of cytokines, cytokine inhibitors, and acute-phase proteins following anti-TNF-alpha therapy in rheumatoid arthritis J. Immunol. 163:1521-1528

22. Kishimoto, T., Taga, T., and Akira, A. 1994. Cytokine signal transduction. Cell.76:253-262.

23. Lockslay, R.M., Killeen, N., and Leonardo, M.J. 2001. The TNF and TNF receptor superfamilies: integrating mammalian biology. Cell. 104:487-501.

24. Firestein, G.S., and Manning, A.M. 1999. Signal transduction and transcription factors in rheumatic diseases. Arthritis Rheum. 42:609-621.

25. Chu, C.Q., Field, M., Feldmann, M., and Maini, R.N. 1991. Localization of tumor necrosis factor alpha in synovial tissues and at the cartilage-pannus junction in patients with rheumatoid arthritis. Arthritis Rheum 34:1125-1132.

26. Ulfgren, A.K., Lindblad, S., Klareskog, L., Anderson, J., and Anderson, U. 1995. Detection of cytokine producing cells in the synovial membrane from patients with rheumatoid arthritis. Ann. Rheum. Dis. 54:654-661.

27. Marinova-Mutafchieva, L., et al. 1997. Dynamics of proinflammatory cytokine expression in the joints of mice with collagen-induced arthritis (CIA). Clin. Exp. Immunol. 107:507-512.

28. Okada, Y., Takeuchi, N., Tomita, K., Nakanishi, I., and Nagase, H. 1989. Immunolocalization of matrix metalloproteinase 3 (stromelysin) in rheumatoid synovioblasts (B cells): correlation with rheumatoid arthritis. Ann. Rheum. Dis. 48:645-653.

29. Tanaka, J., et al. 2000. Lipopolysaccharide-induced HIV-1 expression in transgenic mice is mediated by tumor necrosis factor-alpha and interleukin-1, but not by interferon-gamma nor interleukin-6. AIDS 14:1299-1307.

30. Feldmann, M., and Maini, R.N. 2001. Anti-TNF alpha therapy of rheumatoid arthritis: what have we learned? Annu. Rev. Immunol. 19:163-196.

31. Persson, S., Mikulowska, A., Narula, S., O'Garra, A., and Holmdahl, R. 1996. Interleukin-10 suppresses the development of collagen type II-induced arthritis and ameliorates sustained arthritis in rats. Scand. J. Immunol. 44:607-614.

32. Walmsley, M., et al. 1996. Interleukin-10 inhibition of the progression of established collagen-induced arthritis. Arthritis Rheum. 39:495-503.

33. Katsikis, P.D., Chu, C.Q., Brennan, F.M., Maini, R.N., and Feldmann, M. 1994. Immunoregulatory role of interleukin 10 in rheumatoid arthritis. J. Exp. Med. 179:1517-1527.

34. Asseman, C., Mauze, S., Leach, M.W., Coffman, R.L., and Powrie, F. 1999. An essential role for interleukin 10 in the function of regulatory $\mathrm{T}$ cells that inhibit intestinal inflammation. J. Exp. Med. 190:995-1004.

35. Goudy, K.S., et al. 2003. Systemic overexpression of IL-10 induces $\mathrm{CD} 4{ }^{+} \mathrm{CD} 25^{+}$cell populations in vivo and ameliorates type 1 diabetes in nonobese diabetic mice in a dose-dependent fashion. J. Immunol. 171:2270-2278

36. Naka, T., Nishimoto, N., and Kishimoto, T. 2002. The paradigm of IL-6: from basic science to medicine [review]. Arthritis Res. 4(Suppl. 3):S233-S242.

37. Pasare, C., and Medzhitov, R. 2003. Toll pathway-dependent blockade of $\mathrm{CD} 4^{+} \mathrm{CD} 25^{+} \mathrm{T}$ cellmediated suppression by dendritic cells. Science. 299:1033-1036

38. Morgan, M.E., et al. 2003. CD25+ cell depletion hastens the onset of severe disease in collagen-induced arthritis. Arthritis Rheum. 48:1452-1460.

39. Wu, A.J., Hua, H., Munson, S.H., and McDevitt, H.O. 2002. Tumor necrosis factor-alpha regulation of $\mathrm{CD} 4{ }^{+} \mathrm{CD} 25^{+} \mathrm{T}$ cell levels in NOD mice. Proc. Natl. Acad. Sci. U. S. A. 99:12287-12292.

40. Ortmann, R.A., and Shevach, E.M. 2001. Susceptibility to collagen-induced arthritis: cytokine-mediated regulation. Clin. Immunol. 98:109-118.

41. Joosten, L.A., et al. 1997. Role of interleukin-4 and interleukin-10 in murine collagen-induced arthritis. Protective effect of interleukin-4 and interleukin-10 treatment on cartilage destruction. Arthritis Rheum. 40:249-260.

42. Dayer, J.M., and Burger, D. 1999. Cytokines and direct cell contact in synovitis: relevance to therapeutic intervention. Artbritis Res. 1:17-20.

43. Miossec, P. 2003. Interleukin-17 in rheumatoid arthritis: if $\mathrm{T}$ cells were to contribute to inflammation and destruction through synergy. Arthritis Rheum. 48:594-601.

44. Roosnek, E., and Lanzavecchia, A. 1991. Efficient and selective presentation of antigen-antibody complexes by rheumatoid factor B cells. J. Exp. Med. 173:487-489.

45. Mageed, R.A., Borretzen, M., Moyes, S.P., Thompson, K.M., and Natvig, J.B. 1997. Rheumatoid factor autoantibodies in health and disease. Ann. N. Y. Acad. Sci. 815:296-311.

46. Dalton, D.K., et al. 1993. Multiple defects of immune cell function in mice with disrupted interferongamma genes. Science. 259:1739-1745.

47. Noben-Trauth, N., Kohler, G., Burki, K., and Ledermann, B. 1996. Efficient targeting of the IL-4 gene in a BALB/c embryonic stem cell line. Transgenic Res. 5:487-491.

48. Hori, S., Nomura, T., and Sakaguchi, S. 2003. Control of regulatory $\mathrm{T}$ cell development by the transcription factor Foxp3. Science. 299:1057-1061.

49. Sakaguchi, S., and Sakaguchi, N. 1990. Thymus and autoimmunity: Capacity of the normal thymus to produce pathogenic self-reactive $\mathrm{T}$ cells and conditions required for their induction of autoimmune disease. J. Exp. Med. 172:537-545. 